\title{
Does the chemical signature of TYC 8442-1036-1 originate from a rotating massive star that died in a faint explosion? ${ }^{\star}$
}

\author{
G. Cescutti ${ }^{1,2}$, M. Valentini ${ }^{3}$, P. François ${ }^{4,5}$, C. Chiappini ${ }^{3}$, E. Depagne ${ }^{6}$, N. Christlieb ${ }^{7}$, and C. Cortés ${ }^{8,9}$ \\ 1 Centre for Astrophysics Research, School of Physics, Astronomy and Mathematics, University of Hertfordshire, College Lane, \\ Hatfield AL10 9AB, UK \\ e-mail: g.cescutti@herts.ac.uk \\ 2 BRIDGCE UK Network (www. bridgce.net), UK \\ 3 Leibniz-Institut für Astrophysik Potsdam (AIP), An der Sternwarte 16, 14482 Potsdam, Germany \\ ${ }^{4}$ GEPI - Observatoire de Paris, 64 avenue de l'Observatoire, 75014 Paris, France \\ 5 Université de Picardie Jules Verne, 33 rue St-Leu, 80080 Amiens, France \\ 6 South African Astronomical Observatory (SAAO), Observatory Road Observatory Cape Town, WC 7925, South Africa \\ 7 Zentrum für Astronomie der Universität Heidelberg, Landessternwarte, Königstuhl 12, 69117 Heidelberg, Germany \\ 8 Departamento de Física, Facultad de Ciencias Básicas, Universidad Metropolitana de la Educación, Av. José Pedro Alessandri 774, \\ 7760197 Nuñoa, Santiago, Chile \\ 9 Millennium Institute of Astrophysics (MAS), 7820436 Macul, Santiago, Chile
}

Received 13 June 2016 / Accepted 10 August 2016

\begin{abstract}
Context. We have recently investigated the origin of chemical signatures observed in Galactic halo stars by means of a stochastic chemical evolution model. We found that rotating massive stars are a promising way to explain several signatures observed in these fossil stars.

Aims. We discuss how the extremely metal-poor halo star TYC 8442-1036-1, for which we have now obtained detailed abundances from VLT-UVES spectra, fits into the framework of our previous work.

Methods. We applied a standard one-dimensional (1D) LTE analysis to the spectrum of this star. We measured the abundances of 14 chemical elements; we computed the abundances for $\mathrm{Na}, \mathrm{Mg}, \mathrm{Ca}, \mathrm{Sc}, \mathrm{Ti}, \mathrm{V}, \mathrm{Cr}, \mathrm{Mn}, \mathrm{Fe}, \mathrm{Ni}$, and $\mathrm{Zn}$ using equivalent widths; we obtained the abundances for $\mathrm{C}, \mathrm{Sr}$, and $\mathrm{Ba}$ by means of synthetic spectra generated by MOOG.

Results. We find an abundance of $[\mathrm{Fe} / \mathrm{H}]=-3.5 \pm 0.13$ dex based on our high-resolution spectrum; this points to an iron content that is lower by a factor of three $(0.5 \mathrm{dex})$ compared to that obtained by a low-resolution spectrum. The star has a $[\mathrm{C} / \mathrm{Fe}]=0.4 \mathrm{dex}$, and it is not carbon enhanced like most of the stars at this metallicity. Moreover, this star lies in the plane $[\mathrm{Ba} / \mathrm{Fe}]$ versus $[\mathrm{Fe} / \mathrm{H}]$ in a relatively unusual position, shared by a few other Galactic halo stars, which is only marginally explained by our past results. Conclusions. The comparison of the model results with the chemical abundance characteristics of this group of stars can be improved if we consider in our model the presence of faint supernovae coupled with rotating massive stars. These results seem to imply that rotating massive stars and faint supernovae scenarios are complementary to each other, and are both required in order to match the observed chemistry of the earliest phases of the chemical enrichment of the Universe.
\end{abstract}

Key words. stars: rotation - stars: massive - Galaxy: evolution - Galaxy: halo - nuclear reactions, nucleosynthesis, abundances stars: abundances

\section{Introduction}

The study of extremely metal-poor (EMP) stars is of fundamental importance to reveal the nucleosynthesis production of the first stars and how they formed and, in general, these stars can be of high value to understand the behaviour of all massive stars. Therefore, the last twenty years have seen incredible efforts by observers worldwide to measure these elusive, far, and faint objects in the Galactic halo, from the pioneering studies of McWilliam et al. (1995) and Ryan et al. (1996) to a new generation of data with $8 \mathrm{~m}$ telescopes as, for example, in Cayrel et al. (2004) and Aoki et al. (2005), to the most recent works, for example, by Yong et al. (2013) and Roederer et al. (2014b).

In our recent work, we have provided an interpretation of the presence in EMP stars of specific chemical signatures by means of stochastic chemical evolution models. Our results supported the scenario in which the first stars that exploded and polluted

\footnotetext{
^ Based on observations made with the ESO Very Large Telescope at Paranal Observatory, Chile (ID 094.B-0781(A); P.I. G. Cescutti).
}

the pristine interstellar medium (ISM) were rotating faster than the present day massive stars. Stellar evolution codes coupled with nuclear reaction chains have shown that this rotation produces mixing in the interior of the stars. This mixing impacts the nucleosynthesis of light elements such as carbon, nitrogen, and oxygen (Hirschi 2007; Meynet et al. 2006), and it also predicts the production of $s$-process elements (Pignatari et al. 2008; Frischknecht et al. 2012, 2016). In this scenario in which the stars were fast rotating, chemical evolution models were able to explain several chemical anomalies observed in the early Universe: the almost solar ratio of $[\mathrm{N} / \mathrm{O}]$ and the increase and spread in the $[\mathrm{C} / \mathrm{O}]$ ratio (Chiappini et al. 2006); the low ${ }^{12} \mathrm{C} /{ }^{13} \mathrm{C}$ ratios (Chiappini et al. 2008); the spread present in the [C/O] and [N/O] ratios (Cescutti \& Chiappini 2010); the primary evolution of Be and B (Prantzos 2012); and the spread between light and heavy neutron capture elements (Cescutti et al. 2013).

In Cescutti \& Chiappini (2014), we also predicted that in this scenario, EMP stars with a supersolar $[\mathrm{Sr} / \mathrm{Ba}]$ ratio were expected to have a barium mainly composed of even isotopes, 
clear signature of an $s$-process pollution by fast rotating massive stars. The observations that we present here were granted in the context of the ESO proposal "Probing the sources of synthesis of neutron capture elements: isotopic ratios of barium in halo stars", meant to verify this thesis. However, the metal-poor halo star TYC 8442-1036-1 was a back-up target and it was selected on the criteria that it was a very metalpoor star in the correct position in the sky, was never observed with high-resolution spectroscopy, and was observed due to bad weather conditions that prevented us from observing our main targets. TYC 8442-1036-1 was not a striking case among the 1777 bright $(9<B<14)$ metal-poor candidates selected from the Hamburg/ESO Survey (HE 2220-4840) by Frebel et al. (2006). Its iron content, measured from analyses of medium-resolution follow-up spectroscopy, was $[\mathrm{Fe} / \mathrm{H}]=-2.91 \mathrm{dex}$. This value is very low, but it is not so impressive in a survey that managed to find HE 1327-2326, the most iron-poor star for almost a decade (Frebel et al. 2005), only recently replaced in this record by SMSS J031300.36-670839.3 (Keller et al. 2014).

Nevertheless, our detailed abundance analysis presents surprises, among which the star is more metal poor than expected by the medium-resolution results. The star also presents a $[\mathrm{C} / \mathrm{Fe}]=$ 0.4 dex and therefore is not a carbon-enhanced star, like most of the stars at this metallicity (Placco et al. 2014); actually, it looks more like a normal star similar to others studied by the First Stars collaboration (Cayrel et al. 2004; Spite et al. 2005, 2006). TYC 8442-1036-1 also shows chemical characteristics in the $[\mathrm{Ba} / \mathrm{Fe}]$ vs. $[\mathrm{Fe} / \mathrm{H}]$ space that are just at the edge of predictions for our best model results in Cescutti \& Chiappini (2014). The case of TYC 8442-1036-1 is relatively rare but not unique, and in fact about other 20 stars are just marginally consistent with our previous modelling; moreover, a few other stars $(\sim 2-3)$ cannot be explained by our model. Among the uncertainties of this model, there is the scenario of the $r$-process events, which is the magneto-rotationally driven scenario in Cescutti \& Chiappini (2014). However, a similar outcome is obtained by assuming different scenarios for the $r$-process events, as in Cescutti et al. (2013) by adopting the electron-capture scenario and in Cescutti et al. (2015) by adopting the neutron stars merger scenario.

In the recent years, another scenario has been investigated to explain the characteristics of the EMP (and ultra metal-poor stars) of the halo. This new scenario does not refer to characteristics of the stars during their lives, but rather on their explosions (Cooke \& Madau 2014). In fact, they investigate the impact of a variations in the explosion energy of the primordial supernovae (SNe), as suggested by Tominaga et al. (2007) in the scenario of the faint $\mathrm{SNe}$. The faint $\mathrm{SNe}$ produce less Fe compared to normal $\mathrm{SNe}$, which impacts the results of a stochastic chemical evolution model for the early Universe. We decided to investigate this scenario in this work to see the impact on the neutron capture elements not considered in previous studies, and to compare its results to the findings provided by the scenario of rotating massive stars. Moreover, the different explosion energy does not impact the production of the studied chemical elements determined by fast rotation. Therefore, the two scenarios can be complementary and we show results in which the two scenarios are coupled; these results can represent a solution to the class of objects with chemical characteristics similar to TYC 8442-1036-1.

\section{Observations and data reduction}

The observations were performed using UVES high-resolution spectrograph (Dekker et al. 2000) in slit mode, mounted at the
UT2 Kueyen Telescope at the ESO Paranal Observatory (Chile). We adopted the standard R530 set-up (Red Arm only, Cross Disperser 3, and centred at $520 \mathrm{~nm}$ ), covering the wavelength interval 4140-6210 ̊ with a resolving power of $R \sim 100000$.

The spectrum was acquired on the night of 4 October 2014, adopting an exposure time of $20 \mathrm{~min}$. The resulting spectrum has an average $\mathrm{S} / \mathrm{N}$ of 100 (from 80 at $4200 \AA$ to 150 at $\lambda>5500 \AA$ ). Details of the observation are summarised in Table 1.

The spectrum was reduced using the ESO standard pipeline for UVES. The radial velocity (RV) was measured from the $\mathrm{H}-\beta$ line. The RV correction was applied using the standard IRAF package $R$ Vcorrect. The correction was confirmed by the subsequent comparison with the wavelengths of $\mathrm{Fe}$ and $\mathrm{Ca}$ lines.

\section{Atmospheric parameters and abundances}

We use stellar model atmospheres interpolated from the grid of one-dimensional (1D) MARCS models (Gustafsson et al. 2008) and performed the analysis using a recent version of the spectral line analysis code MOOG ${ }^{1}$. Effective temperatures $\left(T_{\text {eff }}\right)$ and microturbulent velocities $\left(\xi_{t}\right)$ are derived by requiring that abundances derived from Fe I lines showed no trend with the excitation potential and line strength. The $\log (g)$ is derived by requiring that the $\mathrm{Fe}$ abundance derived from Fe I lines matches that derived from Fe II lines. This analysis technique is a standard analysis and very similar to that adopted in Roederer et al. (2014a). Our calculations assume local thermodynamical equilibrium (LTE). The reference solar abundances used in this work are taken from Asplund et al. (2009).

The line list adopted in this work was created starting from the line adopted in Hill et al. (2002), Roederer (2013), and Ural et al. (2015) supplemented by lines taken directly from VALD3 (Kupka et al. 2000). The line list was constructed to avoid blends with other atomic lines and molecular bands as much as possible. The EW of the lines was measured using the ARES (Automatic Routine for line Equivalent widths in stellar Spectra; Sousa et al. 2007). In Table A.1, we report the wavelengths, excitation energy of the lower energy level, oscillator strength, and EW for the considered lines. A fraction of the considered lines was measured using standard IRAF routine: no bias or significant differences between the automatically measured EWs and those determined with the automatic routine was detected. The final atmospheric parameters derived are $T_{\text {eff }}=4800 \mathrm{~K}, \log (g)=1.71 \mathrm{dex}$ and $[\mathrm{Fe} / \mathrm{H}]=-3.50 \mathrm{dex}$ with $\xi_{t}=1.71 \mathrm{~km} \mathrm{~s}^{-1}$.

For some abundances in Table 2 the standard variation $\left(\sigma_{\epsilon}\right.$, Col. 5) involving small numbers of lines (Col. 6) is implausibly small. Therefore, for the abundances measured with less than five lines we assume as random error (Col. 7) the largest standard variation obtained for the remaining abundances ( $0.11 \mathrm{dex}$ for Fe II). The total error is obtained by quadratically adding this updated random error with the systematic error. To calculate the systematic errors, we re-computed the abundances, varying the model atmosphere considering these uncertainties: $\Delta T_{\text {eff }} \pm 100 \mathrm{~K}, \Delta \log (g) \pm 0.3 \mathrm{dex}, \Delta[\mathrm{Fe} / \mathrm{H}] \pm 0.3 \mathrm{dex}$, and $\Delta \xi_{t} \pm$ $0.3 \mathrm{~km} \mathrm{~s}^{-1}$. This method of calculating the systematic errors and uncertainties adopted is based on the recent work on EMP halo stars by Yong et al. (2013); however a similar method and values are also used in the work by Roederer et al. (2014a). The quadratic sum of these variations is reported in Table 2 .

We underline that the analysis performed in this work was made under the assumption of 1D LTE. Assuming LTE, means

http://www . as. utexas. edu/ chris/moog.html 
Table 1. Observing log.

\begin{tabular}{|c|c|c|c|c|c|c|c|c|c|}
\hline Object & $\begin{array}{c}\text { RA } \\
(\mathrm{J} 2000.0)\end{array}$ & $\begin{array}{c}\text { Dec } \\
(\mathrm{J} 2000.0)\end{array}$ & $\begin{array}{c}B \\
(\mathrm{mag})\end{array}$ & $\begin{array}{c}V \\
(\mathrm{mag})\end{array}$ & $\begin{array}{l}\text { Exposure } \\
\text { time }(\mathrm{s})\end{array}$ & $S / N$ & $\begin{array}{l}\text { Obs. date } \\
\text { (UT) }\end{array}$ & $\begin{array}{c}V_{r} \\
\left(\mathrm{~km} \mathrm{~s}^{-1}\right) \\
\end{array}$ & $\begin{array}{c}\text { Error } \\
\left(\mathrm{km} \mathrm{s}^{-1}\right)\end{array}$ \\
\hline TYC 8442-1036-1 & 222323.3 & -482450.9 & 11.71 & 11.10 & 1200 & $>80$ & 4th October 2014 & 90.6 & 0.6 \\
\hline
\end{tabular}

Table 2. Abundances of chemical elements in TYC 8442-1036-1.

\begin{tabular}{crrrrccccc}
\hline \hline Chemical elements & $\epsilon(X)$ & {$[\mathrm{X} / \mathrm{H}]$} & {$[\mathrm{X} / \mathrm{Fe}]$} & $\sigma_{\epsilon(x)}$ & $\mathrm{N}$. lines & $\begin{array}{c}\text { Random } \\
\text { error }\end{array}$ & $\begin{array}{c}\text { Systematic } \\
\text { error }\end{array}$ & $\begin{array}{c}\text { Total } \\
\text { error }\end{array}$ & Corrections non-LTE \\
\hline C (CH) & 5.33 & -3.10 & 0.40 & - & synth & $(0.20)$ & $(0.12)$ & 0.23 & - \\
Na I & 2.48 & -3.76 & -0.26 & 0.01 & 2 & 0.11 & 0.03 & 0.11 & -0.06 \\
Mg I & 4.73 & -2.88 & 0.62 & 0.05 & 3 & 0.11 & 0.01 & 0.11 & 0.10 \\
Ca I & 3.14 & -3.20 & 0.30 & 0.07 & 11 & 0.07 & 0.05 & 0.08 & - \\
Sc II & -0.27 & -3.42 & 0.08 & 0.06 & 6 & 0.06 & 0.11 & 0.12 & - \\
Ti I & 1.67 & -3.27 & 0.23 & 0.07 & 8 & 0.07 & 0.03 & 0.08 & - \\
Ti II & 1.65 & -3.29 & 0.21 & 0.10 & 23 & 0.10 & 0.12 & 0.15 & - \\
V I & 0.42 & -3.51 & -0.01 & - & 1 & 0.11 & 0.04 & 0.12 & - \\
Cr I & 1.63 & -4.01 & -0.51 & 0.09 & 4 & 0.11 & 0.03 & 0.11 & - \\
Mn I & 1.79 & -3.64 & -0.14 & 0.07 & 2 & 0.11 & 0.03 & 0.11 & - \\
Fe I & 4.00 & -3.50 & - & 0.06 & 86 & 0.06 & 0.11 & 0.13 & 0.10 \\
Fe II & 4.00 & -3.50 & - & 0.11 & 11 & 0.11 & 0.09 & 0.14 & - \\
Ni I & 3.03 & -3.19 & 0.31 & - & 1 & 0.11 & 0.04 & 0.12 & - \\
Zn I & 1.36 & -3.20 & 0.30 & 0.09 & 2 & 0.11 & 0.05 & 0.12 & - \\
Sr II & -1.43 & -4.30 & -0.80 & - & synth & $(0.20)$ & $(0.12)$ & 0.23 & - \\
Ba II & -1.72 & -3.90 & -0.40 & - & synth & $(0.20)$ & $(0.12)$ & 0.23 & - \\
\hline
\end{tabular}

Notes. For the abundance values in Col. 4, we use the solar abundances by Asplund et al. (2009).

to imply that the energy distribution is performed only by particle collision. This assumption stops being true close to the stellar surface (Bergemann \& Nordlander 2014). TYC 8442-1036-1 is a giant and EMP star and the non-LTE effects can be important in particular for $\mathrm{Na}, \mathrm{Mg}$, and Fe. In Table 2, we report non-LTE corrections for these elements taken from literature to indicate the expected non-LTE effects. We find a variation up to +0.10 for $\mathrm{Mg}$ on two of the three lines considered here (see Osorio \& Barklem 2016). However, following Merle et al. (2011), the corrections for $\mathrm{Mg}$ abundance are +0.19 dex for the line at $4703 \AA$ and 0.25 dex for the line at $5528 \AA$. An intermediate result is obtained with the corrections by Mashonkina (2013). Also, we do not take into account the line at $4571 \AA$ for which the correction could have been higher (up to 0.3 dex). For an estimate of the non-LTE correction of $\mathrm{Na}$, we provide the corrections calculated by the online database INSPECT ${ }^{2}$; the corrections for $\mathrm{Na}$ in this database are from Lind et al. (2011). Finally, we expect a non-LTE corrections for our Fe I of 0.1 dex $(0.2$ dex at maximum), based on Lind et al. (2012). The abundance of Fe II is not expected to be affected by the non-LTE corrections and its value is still compatible with the estimate value of Fe I with non-LTE corrections. The corrections for non-LTE effects can be important for Mn and Cr (Bergemann \& Gehren 2008; Bergemann \& Cescutti 2010); however, concerning Mn, recent results obtained by Sneden et al. (2016) can challenge the impact of non-LTE corrections. In Sect. 6 we present figures with the abundances of TYC 8442-1036-1 and other observational data taken from literature. In these figures, we use the abundances without non-LTE corrections, since considering these corrections does not alter our conclusions and most of the other data do not consider these corrections. 2 http://inspect.coolstars19.com, A database for Interactive
NLTE Spectroscopy of late-type stars.
Abundances of $\mathrm{C}, \mathrm{Sr}$, and $\mathrm{Ba}$ are derived with the spectral synthesis module of MOOG. The abundances of the elements are iteratively varied until the synthetic spectrum matched the observed spectrum by visual inspection. The macro-turbulent broadening is determined using a Gaussian representing the combined effects of the instrumental profile, atmospheric turbulence, and stellar rotation. The width of this Gaussian is estimated during the spectrum synthesis fitting, and the abundances are thus (slightly) sensitive to the adopted broadening. The lines of $\mathrm{Ba}$ II are affected by hyperfine splitting and also by isotopic splitting. Therefore, the $\mathrm{Ba}$ abundances are computed assuming the McWilliam (1998) $r$-process isotopic composition and hyperfine splitting. In Fig. 1, we compare the differences arising in the shape of the synthetic line of $\mathrm{Ba}$ at $4554 \AA$ when two different isotopic compositions, a solar composition and a typical $s$-process composition, are adopted. We note that the final abundance obtained from the lines $4554 \AA$ and $4914 \AA$ are about 0.4 dex lower, taking the hyperfine splitting and isotopic splitting into account. On the contrary, the abundance calculated on the lines $6110 \AA$ and $5883 \AA$ are in good agreement with the abundances measured from their EWs. This was expected, given the lower impact of the splitting in the latter lines. Also for the measurement of $\mathrm{Sr}$ abundance we decided to use the synthetic analysis (see Fig. 2). The $\mathrm{C}$ abundance was measured in a similar way from analysis of the band of the A-X electronic transitions of the $\mathrm{CH}$ molecule. Figure 3 shows a comparison of the synthetic spectrum with the data in the range 4303-4307 $\AA$. The random abundance errors obtained with synthetic spectra $(\mathrm{C}, \mathrm{Sr}$, and $\mathrm{Ba}$ ) are assumed to be $0.2 \mathrm{dex}$; in fact, for the three analysed elements the observed spectrum is inside this variation of the synthetic spectrum (see Figs. 2 and 3). We also assumed for the systematic errors of these abundance $0.12 \mathrm{dex}$, the maximum systematic error computed among the other elements, for a total error of 0.23 dex. 


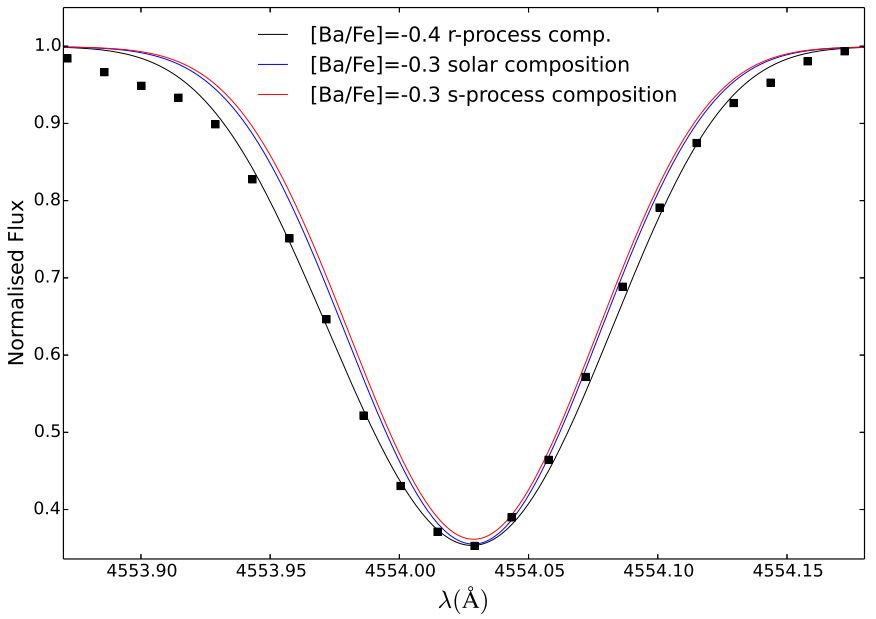

Fig. 1. Fit of the Ba line at $4554 \AA$. The observed spectrumi is represented by dots, the synthetic spectra by lines. We present the different results obtained using different isotopic compositions for $\mathrm{Ba}$ : $r$-process composition, s-process composition, and solar composition. We obtained different best abundances for the different compositions.

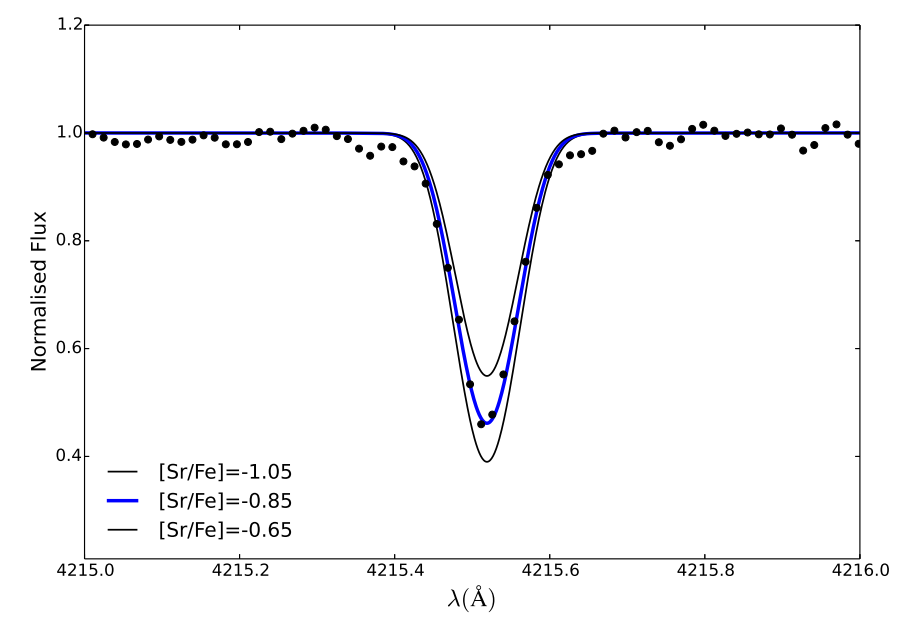

Fig. 2. Fit of the Sr line at $4607 \AA$ A. Dots: observed spectrum, lines: synthetic spectra. The synthetic spectra are calculated for 3 different abundances of Sr. The intermediate abundance was taken as the best value for the $\mathrm{Sr}$ abundance measured in this star.

\section{Chemical evolution model}

The chemical evolution model used is the same as in Cescutti \& Chiappini (2014), which is based on the stochastic model developed in Cescutti (2008), but with a different treatment of the gas flows, following the homogeneous model of Chiappini et al. (2008). The halo is assumed to consist of many independent regions, each with the same typical volume, and each region does not interact with the others. Accordingly, the dimensions of the volume are expected to be large enough to allow us to neglect the interactions between different volumes, at least as a first approximation. For typical ISM densities, a supernova remnant becomes indistinguishable from the ISM, that is, it merges with the ISM before reaching $\sim 50 \mathrm{pc}$ (Thornton et al. 1998); therefore, we decided to have a typical volume with a radius of roughly $90 \mathrm{pc}$. The dimension of this volume is the same as in our previous works adopting a stochastic model for the Galactic halo (Cescutti 2008; Cescutti \& Chiappini 2010, 2014; Cescutti et al. 2013, 2015). The number of assumed volumes to ensure a good statistics in our previous models was 100; however given the variation we implement here for the iron yields

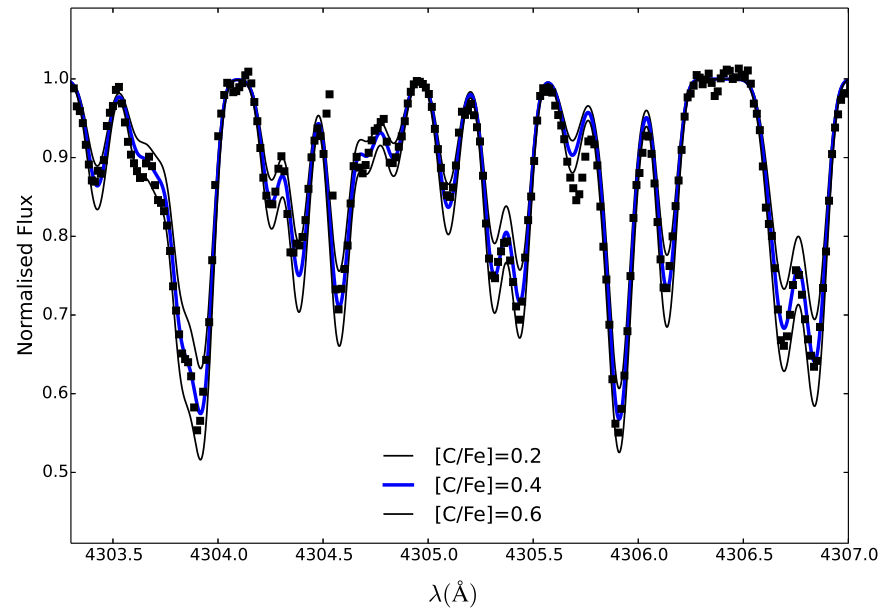

Fig. 3. Fit of $\mathrm{CH}$ lines of the $G$ band in TYC 8442-1036-1. Dots: observations; lines: synthetic spectra computed for the abundances indicated.

(see next section), the new models are based on the results of 1000 volumes. We did not use larger volumes because they would produce more homogeneous results; in fact, in larger volumes the model would predict more $\mathrm{SNe}$ events and a mixture of enrichments that would decrease the maximum spread possible for the set of yields used. Knowing the mass that is transformed into stars in a time step (hereafter, $M_{\text {stars }}^{\text {new }}$ ), we assigned the mass to one star with a random function, weighted according to the initial mass function (IMF) of Scalo (1986) in the range between 0.1 and $100 M_{\odot}$. We then extracted the mass of another star and repeated this cycle until the total mass of newly formed stars exceeded $M_{\text {stars }}^{\text {new }}$. In this way, $M_{\text {stars }}^{\text {new }}$ is the same in each region at each time step, but the total number and mass distribution of the stars are different. We thus know the mass of each star contained in each region, when it is born and when it will die, assuming the stellar lifetimes of Maeder \& Meynet (1989). At the end of its lifetime, each star enriches the ISM with its newly produced chemical elements and with the elements locked in that star when it was formed, excluding the fractions of the elements that are permanently locked into the remnant. As shown in Cescutti et al. (2013), our model is able to reproduce the MDF measured for the halo by Li et al. (2010). This comparison shows that the timescale of enrichment of the model is compatible with that of the halo stars in the solar vicinity. Moreover, our model predicts a small spread for the $\alpha$-elements $\mathrm{Ca}$ and $\mathrm{Si}$, which is compatible with the observational data.

\section{Modelling the nucleosynthesis}

\subsection{Stellar yields for $\mathrm{Fe}$}

Our goal is to explore the impact on the chemical evolution model of the scenario in which massive stars do not always explode as SNe II with a standard energy of $10^{51} \mathrm{erg}$, but they also explode with fainter explosions, as observationally motivated by Moriya et al. (2010).

At present, the mechanism of the explosion of a $\mathrm{SNe}$ II (see Janka et al. 2012), as well as possible connection between mass and explosion energy for SNe, are not fully understood. Therefore, in the nucleosynthesis results, the explosions are not obtained from first principles, and they must be tuned in some way, typically given final kinetic energy of the ejecta or a given amount of Fe ejected (see Chieffi \& Limongi 2013; Woosley \& Weaver 1995; Nomoto et al. 2006). 
In our model rather than stochastically selecting an explosion energy and calculating the Fe ejected, we directly vary the Fe ejected. In particular, we decided to assume, for the production of iron in massive stars $\left(8-80 M_{\odot}\right)$, a distribution of yields that goes from almost zero, $10^{-5} M_{\odot}$ in the case of the faintest explosions, to $0.2 M_{\odot}$. In this range, any value has the same probability to be randomly chosen, so on average a massive star enriches the ISM with $0.1 M_{\odot}$ of iron; in this way, the mean chemical evolution of $\mathrm{Fe}$ is preserved.

These assumptions are crude, but given the complexity connected to the process of the explosion of a SNe II, we decided to keep our assumptions as simple as possible. With this hypothesis, we can check in our stochastic model the impact of the presence of a distribution of energies from faint $\mathrm{SNe}$ to normal SNe. In Sect. 6, we compare the results obtained in Cescutti \& Chiappini (2014) with our standard assumptions for Fe: the solar metallicity yields of Woosley \& Weaver (1995). In all models, we considered the $\mathrm{SNe}$ Ia enrichment, as in Cescutti et al. (2006).

\subsection{Stellar yields for $C$}

For carbon, we present the results with two set of yields for the massive stars and low-intermediate mass stars. We chose these sets to visualise the difference between the carbon production in rotating and non-rotating stellar evolution models as follows:

- Rotating yields, the set of yields of the model as described in Cescutti \& Chiappini (2010), based on the yields by Meynet \& Maeder (2002) for $z \geq 10^{-5}$, and on the total yields by Hirschi (2007) for $z=10^{-8}$. For low-intermediate mass stars, we adopt the stellar yields by Meynet \& Maeder (2002).

- Non-rotating yields, the carbon yields calculated by Woosley \& Weaver (1995), which is a set of yields frequently adopted in chemical evolution studies, but without rotation. For the low-intermediate mass stars we assume the yields by van den Hoek \& Groenewegen (1997).

The two set of yields for carbon do not originate from the same group and/or stellar evolution code, so it is possible that systematic effects also produce differences between them and not only the rotation. Nevertheless, we confirm that yields from the Geneve group without rotation produce less carbon compared to the rotating yields, similar to the non-rotating yields assumed here.

\subsection{Stellar yields for $\mathrm{Ba}$ and $\mathrm{Sr}$}

For barium and strontium, we use the nucleosynthesis of the $M R D+s B 2$ model described in Cescutti \& Chiappini (2014) for all the models with rotating massive stars. These elements can be produced in this model by both the $s$-process and the $r$-process in massive stars. The assumed $r$-process scenario follows the idea described in Winteler et al. (2012) and recently confirmed by Nishimura et al. (2015), where a small percentage of massive stars end their lives as magneto-rotationally driven (MRD) $\mathrm{SNe}$. To implement this scenario into our chemical evolution model, we randomly select $10 \%$ of all the simulated massive stars and we assume that these massive stars generate an $r$ process event at the end of their lives. We have no prediction of the ejected mass in each $r$-process event. On these grounds, we assume that the MRD scenario produces the same amount of $\mathrm{Ba}$ in a stellar generation as the EC+s model (Cescutti et al. 2013); these empirical yields were obtained as the simplest array that is able to reproduce the observed trend in Galactic halo stars of increasing $[\mathrm{Ba} / \mathrm{Fe}]$ with metallicity. In this scenario, we also take into account the possibility that the amount of $r$-process material ejected is not constant (for details on the variation see Cescutti \& Chiappini 2014). The presence or absence of rotation does not influence the $r$-process production in our set of yields. The contribution by the $s$-process in rotating massive stars is assumed as in the fs-model of Cescutti et al. (2013), where we considered the stellar yields obtained by Frischknecht et al. (2012). The barium and strontium produced by the $s$-process is only barely affected by the SNe II explosion, and therefore it is relatively safe to consider a variation of iron without changing these yields. It may be not the case for the Ba $r$-process production, and we comment on this in Sect. 6. In the non-rotating model there is no $s$-process production of $\mathrm{Ba}$ and $\mathrm{Sr}$ from rotating massive stars. However, in all the models, we consider the $s$-process contribution from stars in the mass range $1.3-3 M_{\odot}$ by implementing the yields by Cristallo et al. $(2009,2011)$. We underline that this production channel only affects the model results at moderate metallicity $([\mathrm{Fe} / \mathrm{H}] \sim-1.5 \mathrm{dex})$.

\section{Results}

\subsection{Results for $\mathrm{Ba}$}

In Fig. 4, we show the results of three models for $[\mathrm{Ba} / \mathrm{Fe}]$ versus $[\mathrm{Fe} / \mathrm{H}]$. Two models take rotating massive stars into account. The first model, which we call the spinning faint scenario, considers the presence of faint $\mathrm{SNe}$ that can produce an almost negligible amount of iron. In the second model, which we call the spinstar scenario (contour plot with dashed line), the $\mathrm{SNe}$ II produce a fixed amount of iron (roughly $0.1 M_{\odot}$ ), following the results by Woosley \& Weaver (1995). In the last model, called the faint SNe scenario (contour plot with solid line), we consider nonrotating massive stars and the presence of faint $\mathrm{SNe}$. The nucleosynthesis yields are summarised in Table 3 . We compare our models to the star analysed here and to a collection of observational data.

The spinstars scenario (which is the same model for $\mathrm{Ba}$ as the model MDR+s B2 realised in Cescutti \& Chiappini 2014) is quite successful because in the space $[\mathrm{Ba} / \mathrm{Fe}]$ vs. $[\mathrm{Fe} / \mathrm{H}]$, the density of its simulated long-living stars matches most of the stars observed in the halo. However, a certain number of objects at extremely low metallicity and low $[\mathrm{Ba} / \mathrm{Fe}]$ are positioned where the model predicts a null density of stars. It was also unable to explain stars at $[\mathrm{Fe} / \mathrm{H}] \sim-3.5 \mathrm{dex}$ at $[\mathrm{Ba} / \mathrm{Fe}] \sim-0.5 \mathrm{dex}$. In particular the star we characterised here is found in this area.

On the other hand, in Fig. 4 the results of the model spinning faint scenario explain, within the observational errors, all the stars at $[\mathrm{Fe} / \mathrm{H}]<-4$ dex. This model also simulates stars at $[\mathrm{Fe} / \mathrm{H}] \sim-3.5 \mathrm{dex}$ at $[\mathrm{Ba} / \mathrm{Fe}] \sim-0.5 \mathrm{dex}$ and is compatible with the star analysed here. The density plot produced by this model shows two bands that move downwards from $[\mathrm{Fe} / \mathrm{H}] \sim$ -6 dex to $[\mathrm{Fe} / \mathrm{H}] \sim-3$ dex. The band at lower $[\mathrm{Ba} / \mathrm{Fe}]$ was enriched by spinstars with an associated SNe II with a low iron production (faint $\mathrm{SNe}$ ). Therefore, this coupling helps to recover the observed stars better at the lowest $[\mathrm{Fe} / \mathrm{H}]$, which are mostly in this lower band. The second band with low $[\mathrm{Fe} / \mathrm{H}]$ but high $[\mathrm{Ba} / \mathrm{Fe}]$ is produced in the model by $r$-process events coupled with weak production of iron. Stars in the region are not observed for $[\mathrm{Fe} / \mathrm{H}]<-4$ dex. If this absence is confirmed by future observations, it will provide an additional contraint to the $r$-process events; these events should be associated with a normal production of $\mathrm{Fe}$ and not with faint $\mathrm{SNe}$. This constraint applies in case of single massive stars as progenitors of the $r$-process 

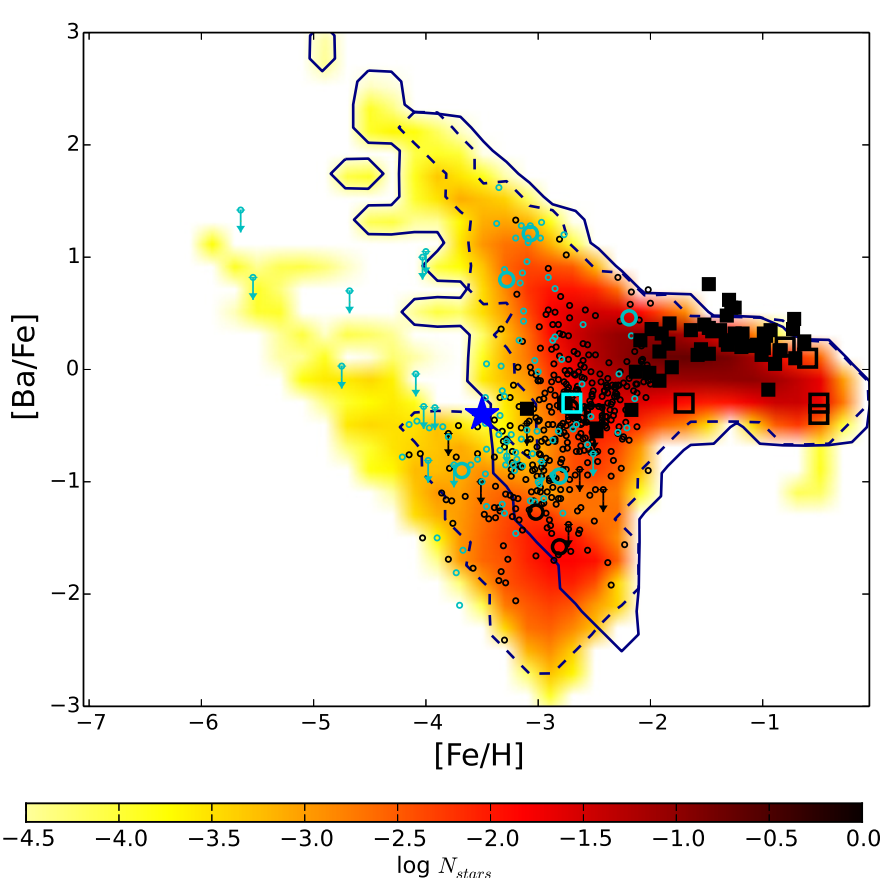

Fig. 4. $[\mathrm{Ba} / \mathrm{Fe}]$ vs. $[\mathrm{Fe} / \mathrm{H}]$. The star analysed here is shown using a blue star; the open small dots are data collected by Placco et al. (2014) and the arrows indicate upper limits; the big open dots are data from Hansen et al. (2015); the solid squares from Hansen et al. (2012); the open squares are taken from Hansen et al. (2016). The cyan symbols refer to carbon-enhanced metal-poor (CEMP) stars without $s$-process enhancement, adopting the criterion $[\mathrm{C} / \mathrm{Fe}]>0.7$ for carbon enhancement, as in Placco et al. (2014); the black symbols are for normal stars. We do not plot stars with carbon and $s$-process enhancement (CEMPs). The colour-coded surface density plot presents the density of longliving stars for the model of spinning faint SNe. The contour plot with the dashed line shows the results for the model of spinstars, the contour plot with the solid line shows the results for the model of faint $\mathrm{SNe}$.

Table 3. Nucleosynthesis prescriptions adopted for the 3 models.

\begin{tabular}{ccc}
\hline \hline Models Figs. 4-6 & Yields for Fe & Yields for Ba and C \\
\hline spinstars scenario & standard & rotating \\
faint SNe scenario & with variance & non-rotating \\
spinning faint scenario & with variance & rotating \\
\hline
\end{tabular}

events, whereas in the case of the merger scenario of neutron stars, it should be applied to at least one of the two massive progenitor stars.

In the model faint SNe scenario, we do not consider the production of $s$-process by massive stars. The results of this model are only marginally consistent with the abundances measured in TYC 8442-1036-1. Considering non-LTE corrections for our star increases its agreement with the faint SNe scenario; however, this model still fails to reproduce the stars located in the band at lower $[\mathrm{Ba} / \mathrm{Fe}]$ in the results of the spinning faint scenario.

\subsection{Results for $[\mathrm{Sr} / \mathrm{Ba}]$}

We present in the Fig. 5, the results of the three models for $[\mathrm{Sr} / \mathrm{Ba}]$ versus $[\mathrm{Fe} / \mathrm{H}]$. Similar to the $[\mathrm{Ba} / \mathrm{Fe}]$ case in the previous section, the spinstars scenario model is really successful because in the space $[\mathrm{Sr} / \mathrm{Ba}]$ versus $[\mathrm{Fe} / \mathrm{H}]$, the density of its simulated long-living stars can recover most of the stars observed in the halo. Still, a certain number of objects are located at extremely

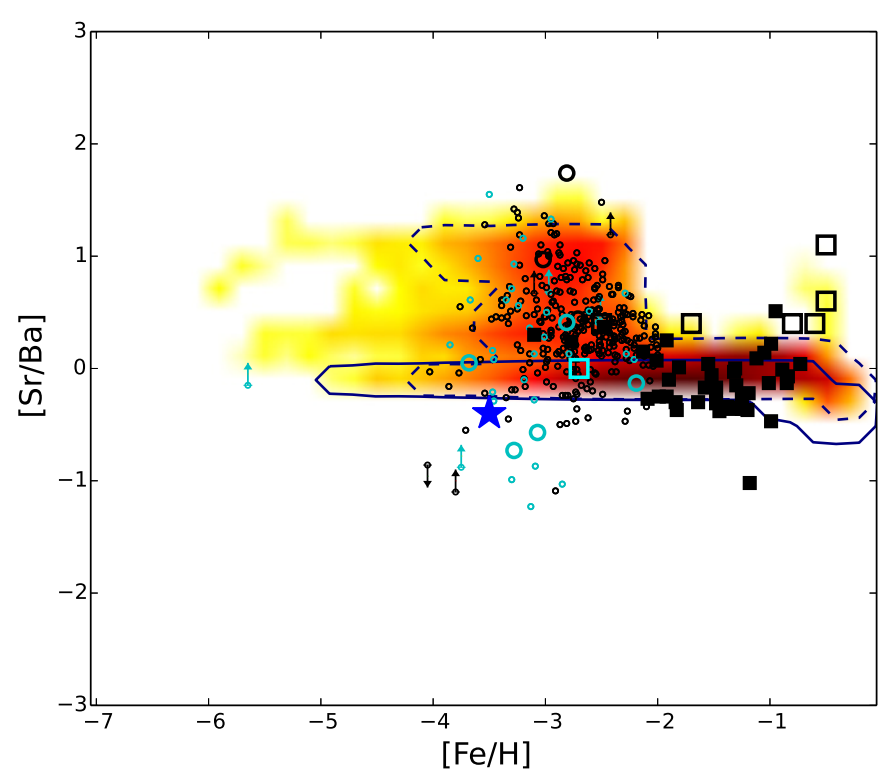

Fig. 5. $[\mathrm{Sr} / \mathrm{Ba}]$ vs. $[\mathrm{Fe} / \mathrm{H}]$. The symbols are the same as in Fig. 4. The colour-coded surface density plot and the contour plots describe the same models as in Fig. 4.

low metallicity, where this model predicts a null density of stars. Again this issue is improved once we also adopt a variation of yield for the iron, as in the case of spinning faint scenario.

Figure 5 also explained why the spinstars $s$-process contribution is essential. Indeed, without this contribution, the faint $\mathrm{SNe}$ scenario cannot reproduce a large number of abundances observed in Galactic stars. We note that in this figure there is also a small fraction of stars that is not compatible with any of the scenarios investigated here. These outliers are located below the $[\mathrm{Sr} / \mathrm{Ba}]$ ratio assumed for the $r$-process events and cannot be reproduced, as the $s$-process is also produced by the spinstars with a $[\mathrm{Sr} / \mathrm{Ba}]>0$. However, their fraction is small and in the $[\mathrm{Sr} / \mathrm{Ba}]$ plot the errors of both chemical element abundances should be considered; therefore a substantial portion of these outliers are still compatible within the errors with the model results and TYC 8442-1036-1 is in this group. Finally, the carbon-enhanced metal-poor (CEMP) stars in this group could be originated in a binary system, therefore they show a mild enhancement due to the pollution of $s$-process material from the companion star. If this possible scenario is taken into account only two EMP stars are outliers compared to our models, which is an excellent result.

\subsection{Results for $C$}

Going towards extremely low metallicity, an increasing number of stars are carbon rich and belong to the category of the CEMP-no stars. In Beers \& Christlieb (2005), a metal-poor star is a CEMP star if $[\mathrm{C} / \mathrm{Fe}]>1$. If there is no excess of $s$-process $([\mathrm{Ba} / \mathrm{Fe}]<1)$ the star belongs to the CEMP-no category, and otherwise it belongs to the CEMP-s class. However, the star we analysed does not belong to the CEMP-no category, as it is only slightly carbon enhanced. Therefore, we decided to investigate how our spinning faint $\mathrm{SNe}$ model behaves in terms of $[\mathrm{C} / \mathrm{Fe}]$ ratio versus $[\mathrm{Fe} / \mathrm{H}]$. The results are shown in Fig. 6. In this figure the density plot represents the results for the spinning faint $\mathrm{SNe}$ scenario, whereas the contour plot with the dashed line shows the results of the spinstars scenario where SNe II produce a fixed amount of iron. The solid line contour plot represents the 


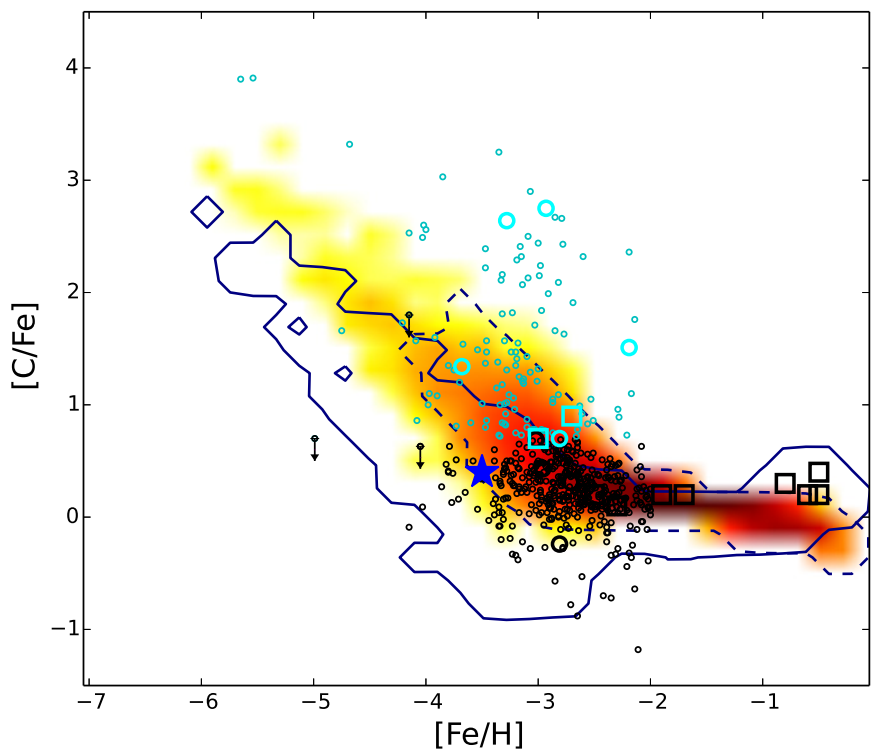

Fig. 6. $[\mathrm{C} / \mathrm{Fe}]$ vs. $[\mathrm{Fe} / \mathrm{H}]$. The symbols are the same as in Fig. 4. The colour-coded surface density plot and the contour plots describe the same models as in Fig. 4.

results assuming non-rotating yields for carbon (see Table 3 for details), and in this model (faint $\mathrm{SNe}$ scenario) we consider a variation in iron yields.

The low production of iron by faint $\mathrm{SNe}$ produces a rise in the $[\mathrm{C} / \mathrm{Fe}]$ ratio towards low metallicity, and this trend is common in both models that consider faint $\mathrm{SNe}$ (spinning faint $\mathrm{SNe}$ scenario and faint $\mathrm{SNe}$ scenario) with and without rotation. However, the model with rotation predicts a density distribution of stars with a $[\mathrm{C} / \mathrm{Fe}]$ ratio about 1 dex higher compared to the nonrotating yields for $[\mathrm{Fe} / \mathrm{H}]<-3$ dex.

The higher production of carbon in the yields with rotation improve the agreement between the model and the data. In fact, a substantial number of Galactic stars is inside the predictions of the faint spinstars model for $-3<[\mathrm{Fe} / \mathrm{H}]<-4$ dex and $[\mathrm{C} / \mathrm{Fe}] \sim$ $1 \mathrm{dex}$, and outside the predictions of the model assuming yields without rotation. The chemical enrichment of the star measured here, is consistent with both rotation and non-rotational models. We also note that the model with rotation cannot explain a portion of stars with lower ratios of $[\mathrm{C} / \mathrm{Fe}]$ that can be reproduced in the model without rotation. This points to a fact that we expected: a distribution of rotational velocities among the massive stars. We underline that it will be also possible in the near future to investigate the most likely distribution of stellar rotation for these low-metallicity massive stars with a set of nucleosynthesis computations in which yields for different rotational velocity are provided. In comparison with the spinning faint SNe scenario, the model with a fixed amount of iron produced by $\mathrm{SNe}$ II (spinstars scenario; dashed contour) cannot reproduce the data for $[\mathrm{Fe} / \mathrm{H}]<-4$ dex. Moreover, a non-negligible number of stars are located just inside the upper edge of the contour for $-4<$ $[\mathrm{Fe} / \mathrm{H}]<-3$, where the results of the model predict a very low density of long-living stars; therefore the model is not fully consistent with the data.

The spinning faint SNe model cannot be considered an exhaustive explanation for the CEMP-no stars. A non-negligible number of the observed CEMP-no stars shows a $[\mathrm{C} / \mathrm{Fe}]$ that is not compatible with the predictions of the model. The carbon present in these objects is in some cases more than 1 dex higher compared to the spinning faint $\mathrm{SNe}$ model results. This class of objects has also been identified in Cooke \& Madau (2014) as super CEMP.

It is likely that the carbon present (at least) in this class of CEMP-no stars was not well mixed in the ISM, before it was locked in these low-mass stars. In Meynet et al. (2010), the chemical signatures present in the three most iron-poor stars known at that time $^{3}$, which are also CEMP-no stars, were explained assuming that these stars were formed (almost) entirely by stellar winds of rotating massive stars. In this scenario the $[\mathrm{C} / \mathrm{Fe}]$ of these stars can be strongly enhanced compared to the results of a standard chemical model, where the stellar enrichment is well mixed with the ISM before forming new stars. In our plots, these stars are present; they belong to the category of stars that lay above our model predictions and are the first three data points starting approximately at $[\mathrm{Fe} / \mathrm{H}]=-6$ dex. The presence of this class of super CEMP, was already noted in Cescutti \& Chiappini (2010), where we could not reconcile our models with a large fraction of the CEMP-no known at that time. The issue was also very clear in the $[\mathrm{C} / \mathrm{O}]$ (and $[\mathrm{N} / \mathrm{O}]$ ) versus $[\mathrm{O} / \mathrm{H}]$ space, where it was possible to neglect the influence of the uncertainties in the production of iron.

Recently, it was also underlined by Maeder \& Meynet (2015) that different subclasses of CEMP-no stars should be considered in the context of formation by stellar winds. These subclasses are probably determined by different degrees of internal mixing during stellar evolution. It will be possible to take all these differences into account only when models with nucleosynthesis covering a broad range of stellar masses, initial metallicities, CNO ratios, rotational velocities, and mass loss rates are available. Therefore, it is not surprising that in the context of a chemical evolution model, where only full mixing with the ISM is considered and just a grid of models for two velocities are available, we cannot fully explain all the CEMP-no stars.

Another possible explanation is that at least a fraction of these CEMP-no stars are the secondary in a binary system. In this scenario, the star presents the pristine composition polluted by AGB material of the primary star. This is the same scenario that is favoured for the CEMP-s, and in this case the absence of strong enrichment of barium can be explained in the framework of classical theoretical yields for AGB stars. The very low metallicity reduces barium production and enhances the production of lead (Cristallo et al. 2011). It is also possible that the evolution of low-mass stars is quite different at extremely low metallicity and can suppress the $s$-process production (Fujimoto et al. 2000; Komiya et al. 2007). As found in Cooke \& Madau (2014), at least three out of five of the stars of this group show evidence that they are binary stars (Starkenburg et al. 2014); however, very recently Hansen et al. (2015) found no compelling relation between binarity and carbon enhancement.

Given the complexity of the observational data, we have shown that the spinning faint $\mathrm{SNe}$ model has successfully recovered the main trend of the data. In fact, the star studied here and a substantial number of extremely metal-poor (CEMP-no and normal) stars can be formed in the framework of normal chemical evolution, if we couple the fast rotating yields and the presence of faint SNe. Moreover, for elements that are not expected to be ejected by stellar winds, such as Ba, we have shown that basically all the observational data available are compatible with the predictions of the spinning faint SNe model.

3 HE 0107-5240 (Christlieb et al. 2002), HE 1327-2326 (Frebel et al. 2005), HE 0557-4840 (Norris et al. 2007); the record is now held by SMSS J031300.36-670839.3 (Keller et al. 2014). 


\section{Conclusions}

We measured 13 chemical elements for TYC 8442-1036-1, a metal-poor star of the Galactic halo, from a high-resolution spectrum. This star belongs to the rare class of EMP stars with $[\mathrm{Fe} / \mathrm{H}]=-3.5 \pm 0.13$ dex, which is 0.5 dex lower than what was previously determined with a medium-resolution spectrum. At this metallicity, most of the stars in the Galactic halo have a $[\mathrm{C} / \mathrm{Fe}]>0.7$ dex and belong to the class of CEMP-no stars. This is not the case for our star which shows just a mild overabundance of carbon $([\mathrm{C} / \mathrm{Fe}]=0.4 \mathrm{dex})$. We have also measured with particular attention its $[\mathrm{Ba} / \mathrm{Fe}]$ ratio, and we find a low abundance of about $[\mathrm{Ba} / \mathrm{Fe}]=-0.4$ dex. This particular abundance pattern was not explained by our previous models for neutron capture elements in the Galactic halo. In our previous work (Cescutti et al. 2013; Cescutti \& Chiappini 2014), we showed that an $r$-process component and the spinstars contribution of $s$-process can account for most of the data in literature. We decided thus to include a variation on the iron yields as well, mimicking the production of iron by faint SNe. The final model, which we call the spinning faint $\mathrm{SNe}$, is able to explain the presence of the most extreme stars in the $[\mathrm{Ba} / \mathrm{Fe}]$ ratio versus $[\mathrm{Fe} / \mathrm{H}]$ space. The comparison of the model with the observational data also indicates that the $r$-process events are not linked to faint $\mathrm{SNe}$ events. Since most of the stars at such a low metallicity appear to be very enhanced in carbon, we also decided to show the results of our model in the $[\mathrm{C} / \mathrm{Fe}]$ ratio versus $[\mathrm{Fe} / \mathrm{H}]$ space. We find that the model is able to explain the chemistry of TYC 8442-1036-1 and also to recover a large number of the CEMP-no stars. However, a non-negligible number still remains not explicable. A scenario to explain these super CEMPno stars is that these stars have been formed (almost) entirely from the material ejected through winds by fast rotating massive stars (Meynet et al. 2010; Maeder \& Meynet 2015).

Acknowledgements. This work was partially supported by the UK Science and Technology Facilities Council (grant ST/M000958/1). We thank the referee for comments that improved the clarity of this paper. G.C. thanks Lorenzo Monaco and Giacomo Beccari for the support during the execution of the observations. G.C. also thanks Raphael Hirschi for his useful suggestions, Marco Limongi for his comments on the iron production, and Sean Ryan and Piercarlo Bonifacio for their advice on the data analysis section. Support for C. Cortés is provided by Proyecto FONDECYT Iniciación a la Investigacion 11150768 and the Chilean Ministry for the Economy, Development, and Tourism's Programa Iniciativa Científica Milenio through grant IC120009, awarded to Millenium Astrophysics Institute.

\section{References}

Aoki, W., Honda, S., Beers, T. C., et al. 2005, ApJ, 632, 611 Asplund, M., Grevesse, N., Sauval, A. J., \& Scott, P. 2009, ARA\&A, 47, 481

Beers, T. C., \& Christlieb, N. 2005, ARA\&A, 43, 531

Bergemann, M., \& Cescutti, G. 2010, A\&A, 522, A9

Bergemann, M., \& Gehren, T. 2008, A\&A, 492, 823

Cayrel, R., Depagne, E., Spite, M., et al. 2004, A\&A, 416, 1117

Cescutti, G. 2008, A\&A, 481, 691

Cescutti, G., \& Chiappini, C. 2010, A\&A, 515, A102

Cescutti, G., \& Chiappini, C. 2014, A\&A, 565, A51

Cescutti, G., François, P., Matteucci, F., Cayrel, R., \& Spite, M. 2006, A\&A, 448, 557

Cescutti, G., Chiappini, C., Hirschi, R., Meynet, G., \& Frischknecht, U. 2013, A\&A, 553, A51
Cescutti, G., Romano, D., Matteucci, F., Chiappini, C., \& Hirschi, R. 2015, A\&A, 577, A139

Chiappini, C., Hirschi, R., Meynet, G., et al. 2006, A\&A, 449, L27 Chiappini, C., Ekström, S., Meynet, G., et al. 2008, A\&A, 479, L9 Chieffi, A., \& Limongi, M. 2013, ApJ, 764, 21

Christlieb, N., Bessell, M. S., Beers, T. C., et al. 2002, Nature, 419, 904

Cooke, R. J., \& Madau, P. 2014, ApJ, 791, 116

Cristallo, S., Straniero, O., Gallino, R., et al. 2009, ApJ, 696, 797

Cristallo, S., Piersanti, L., Straniero, O., et al. 2011, ApJS, 197, 17

Dekker, H., D'Odorico, S., Kaufer, A., Delabre, B., \& Kotzlowski, H. 2000, in Optical and IR Telescope Instrumentation and Detectors, eds. M. Iye, \& A. F. Moorwood, SPIE Conf. Ser., 4008, 534

Frebel, A., Aoki, W., Christlieb, N., et al. 2005, Nature, 434, 871

Frebel, A., Christlieb, N., Norris, J. E., et al. 2006, ApJ, 652, 1585

Frischknecht, U., Hirschi, R., \& Thielemann, F.-K. 2012, A\&A, 538, L2

Frischknecht, U., Hirschi, R., Pignatari, M., et al. 2016, MNRAS, 456, 1803

Fujimoto, M. Y., Ikeda, Y., \& Iben, Jr., I. 2000, ApJ, 529, L25

Gustafsson, B., Edvardsson, B., Eriksson, K., et al. 2008, A\&A, 486, 951

Hansen, C. J., Primas, F., Hartman, H., et al. 2012, A\&A, 545, A31

Hansen, C. J., Nordström, B., Hansen, T. T., et al. 2016, A\&A, 588, A37

Hansen, T., Hansen, C. J., Christlieb, N., et al. 2015, ApJ, 807, 173

Hill, V., Plez, B., Cayrel, R., et al. 2002, A\&A, 387, 560

Hirschi, R. 2007, A\&A, 461, 571

Janka, H.-T., Hanke, F., Hüdepohl, L., et al. 2012, Prog. Theor. Exp. Phys., 2012, 010000

Keller, S. C., Bessell, M. S., Frebel, A., et al. 2014, Nature, 506, 463

Komiya, Y., Suda, T., Minaguchi, H., et al. 2007, ApJ, 658, 367

Kupka, F. G., Ryabchikova, T. A., Piskunov, N. E., Stempels, H. C., \& Weiss, W. W. 2000, Balt. Astron., 9, 590

Li, H. N., Christlieb, N., Schörck, T., et al. 2010, A\&A, 521, A10

Lind, K., Asplund, M., Barklem, P. S., \& Belyaev, A. K. 2011, A\&A, 528, A103

Lind, K., Bergemann, M., \& Asplund, M. 2012, MNRAS, 427, 50

Maeder, A., \& Meynet, G. 1989, A\&A, 210, 155

Maeder, A., \& Meynet, G. 2015, A\&A, 580, A32

Mashonkina, L. 2013, A\&A, 550, A28

McWilliam, A. 1998, AJ, 115, 1640

McWilliam, A., Preston, G. W., Sneden, C., \& Searle, L. 1995, AJ, 109, 2757

Merle, T., Thévenin, F., Pichon, B., \& Bigot, L. 2011, MNRAS, 418, 863

Meynet, G., \& Maeder, A. 2002, A\&A, 390, 561

Meynet, G., Ekström, S., \& Maeder, A. 2006, A\&A, 447, 623

Meynet, G., Hirschi, R., Ekstrom, S., et al. 2010, A\&A, 521, A30

Moriya, T., Tominaga, N., Tanaka, M., et al. 2010, ApJ, 719, 1445

Nishimura, N., Takiwaki, T., \& Thielemann, F.-K. 2015, ApJ, 810, 109

Nomoto, K., Tominaga, N., Umeda, H., Kobayashi, C., \& Maeda, K. 2006, Nucl. Phys. A, 777,424

Norris, J. E., Christlieb, N., Korn, A. J., et al. 2007, ApJ, 670, 774

Osorio, Y., \& Barklem, P. S. 2016, A\&A, 586, A120

Pignatari, M., Gallino, R., Meynet, G., et al. 2008, ApJ, 687, L95

Placco, V. M., Frebel, A., Beers, T. C., \& Stancliffe, R. J. 2014, ApJ, 797, 21

Prantzos, N. 2012, A\&A, 542, A67

Roederer, I. U. 2013, AJ, 145, 26

Roederer, I. U., Cowan, J. J., Preston, G. W., et al. 2014a, MNRAS, 445, 2970

Roederer, I. U., Preston, G. W., Thompson, I. B., et al. 2014b, AJ, 147, 136

Ryan, S. G., Norris, J. E., \& Beers, T. C. 1996, ApJ, 471, 254

Scalo, J. M. 1986, Fund. Cosmic Phys., 11, 1

Sneden, C., Cowan, J. J., Kobayashi, C., et al. 2016, ApJ, 817, 53

Sousa, S. G., Santos, N. C., Israelian, G., Mayor, M., \& Monteiro, M. J. P. F. G. 2007, A\&A, 469, 783

Spite, M., Cayrel, R., Plez, B., et al. 2005, A\&A, 430, 655

Spite, M., Cayrel, R., Hill, V., et al. 2006, A\&A, 455, 291

Starkenburg, E., Shetrone, M. D., McConnachie, A. W., \& Venn, K. A. 2014, MNRAS, 441, 1217

Thornton, K., Gaudlitz, M., Janka, H.-T., \& Steinmetz, M. 1998, ApJ, 500, 95

Tominaga, N., Maeda, K., Umeda, H., et al. 2007, ApJ, 657, L77

Ural, U., Cescutti, G., Koch, A., et al. 2015, MNRAS, 449, 761

van den Hoek, L. B., \& Groenewegen, M. A. T. 1997, A\&AS, 123, 305

Winteler, C., Käppeli, R., Perego, A., et al. 2012, ApJ, 750, L22

Woosley, S. E., \& Weaver, T. A. 1995, ApJS, 101, 181

Yong, D., Norris, J. E., Bessell, M. S., et al. 2013, ApJ, 762, 26 
Appendix A: Additional table

Table A.1. Equivalent width for TYC 8442-1036-1.

\begin{tabular}{|c|c|c|c|c|}
\hline Wavelength $(\AA)$ & Species & L.E.P. $(\mathrm{eV})$ & $\log g f$ & $W_{\lambda}(\mathrm{m} \AA)$ \\
\hline 5889.951 & $\mathrm{Na} \mathrm{I}$ & 0.000 & 0.112 & 89.11 \\
\hline 5895.924 & $\mathrm{Na} \mathrm{I}$ & 0.000 & -0.191 & 69.92 \\
\hline 4351.906 & Mg I & 4.340 & -0.525 & 29.42 \\
\hline 4702.990 & $\operatorname{Mg~I}$ & 4.330 & -0.380 & 43.16 \\
\hline 5528.405 & Mg I & 4.340 & -0.341 & 42.12 \\
\hline 4283.011 & $\mathrm{Ca} \mathrm{I}$ & 1.890 & -0.220 & 22.29 \\
\hline 4318.652 & $\mathrm{Ca} \mathrm{I}$ & 1.900 & -0.210 & 18.21 \\
\hline 4425.437 & $\mathrm{Ca} \mathrm{I}$ & 1.880 & -0.360 & 15.01 \\
\hline 4435.679 & $\mathrm{Ca} \mathrm{I}$ & 1.890 & -0.520 & 8.57 \\
\hline 4454.779 & $\mathrm{Ca} \mathrm{I}$ & 1.900 & 0.260 & 35.86 \\
\hline 5265.556 & $\mathrm{Ca} \mathrm{I}$ & 2.520 & -0.260 & 5.54 \\
\hline 5588.749 & $\mathrm{Ca} \mathrm{I}$ & 2.520 & 0.210 & 12.43 \\
\hline 5857.451 & $\mathrm{Ca} \mathrm{I}$ & 2.930 & 0.230 & 4.99 \\
\hline 6102.723 & $\mathrm{Ca} \mathrm{I}$ & 1.880 & -0.790 & 6.63 \\
\hline 6122.217 & $\mathrm{Ca} \mathrm{I}$ & 1.890 & -0.320 & 17.35 \\
\hline 6162.173 & $\mathrm{Ca} \mathrm{I}$ & 1.900 & -0.090 & 27.52 \\
\hline 4246.822 & Sc II & 0.310 & 0.240 & 83.71 \\
\hline 4314.083 & Sc II & 0.620 & -0.100 & 46.27 \\
\hline 4400.389 & Sc II & 0.610 & -0.540 & 29.74 \\
\hline 4415.557 & Sc II & 0.600 & -0.670 & 23.33 \\
\hline 5031.021 & Sc II & 1.360 & -0.400 & 8.18 \\
\hline 5526.790 & Sc II & 1.770 & 0.030 & 5.78 \\
\hline 4533.241 & Ti I & 0.850 & 0.480 & 16.02 \\
\hline 4534.776 & Ti I & 0.840 & 0.280 & 13.82 \\
\hline 4981.731 & Ti I & 0.840 & 0.500 & 20.44 \\
\hline 4991.065 & Ti I & 0.840 & 0.380 & 16.15 \\
\hline 4999.503 & Ti I & 0.830 & 0.250 & 11.85 \\
\hline 5014.276 & Ti I & 0.810 & 0.110 & 8.80 \\
\hline 5039.957 & Ti I & 0.020 & -1.130 & 4.20 \\
\hline 5064.653 & Ti I & 0.050 & -0.990 & 4.67 \\
\hline 4290.219 & Ti II & 1.160 & -0.930 & 56.93 \\
\hline 4300.049 & Ti II & 1.180 & -0.490 & 61.91 \\
\hline 4337.915 & Ti II & 1.080 & -0.980 & 53.50 \\
\hline 4394.051 & Ti II & 1.220 & -1.770 & 12.88 \\
\hline 4395.033 & Ti II & 1.080 & -0.510 & 73.55 \\
\hline 4395.850 & Ti II & 1.240 & -1.970 & 7.46 \\
\hline 4399.772 & Ti II & 1.240 & -1.220 & 38.05 \\
\hline 4417.719 & Ti II & 1.160 & -1.230 & 42.94 \\
\hline 4418.330 & Ti II & 1.240 & -1.990 & 6.96 \\
\hline 4443.794 & Ti II & 1.080 & -0.700 & 74.70 \\
\hline 4444.558 & Ti II & 1.120 & -2.210 & 10.41 \\
\hline 4450.482 & Ti II & 1.080 & -1.510 & 31.54 \\
\hline 4464.450 & Ti II & 1.160 & -1.810 & 15.35 \\
\hline 4468.507 & Ti II & 1.130 & -0.600 & 68.69 \\
\hline 4470.857 & Ti II & 1.160 & -2.060 & 7.40 \\
\hline 4501.273 & Ti II & 1.120 & -0.760 & 66.05 \\
\hline 4533.969 & Ti II & 1.240 & -0.540 & 67.72 \\
\hline 4563.761 & Ti II & 1.220 & -0.790 & 58.07 \\
\hline 4571.968 & Ti II & 1.570 & -0.230 & 57.79 \\
\hline 4657.200 & Ti II & 1.240 & -2.240 & 4.38 \\
\hline 5129.152 & Ti II & 1.890 & -1.300 & 6.00 \\
\hline 5336.771 & Ti II & 1.580 & -1.630 & 9.19 \\
\hline 5381.015 & Ti II & 1.570 & -1.970 & 5.72 \\
\hline 4379.230 & V I & 0.300 & 0.550 & 5.11 \\
\hline 4254.332 & $\mathrm{Cr} \mathrm{I}$ & 0.000 & -0.110 & 66.85 \\
\hline 4274.796 & Cr I & 0.000 & -0.230 & 60.79 \\
\hline
\end{tabular}

Table A.1. continued.

\begin{tabular}{|c|c|c|c|c|}
\hline Wavelength $(\AA)$ & Species & L.E.P. $(\mathrm{eV})$ & $\log g f$ & $W_{\lambda}(\mathrm{m} \AA)$ \\
\hline 4289.716 & $\mathrm{Cr} \mathrm{I}$ & 0.000 & -0.360 & 55.05 \\
\hline 5409.772 & $\mathrm{Cr} \mathrm{I}$ & 1.030 & -0.720 & 6.65 \\
\hline 4754.042 & Mn I & 2.280 & -0.090 & 5.34 \\
\hline 4823.524 & Mn I & 2.320 & 0.140 & 6.50 \\
\hline 4184.890 & $\mathrm{Fe} \mathrm{I}$ & 2.830 & -0.870 & 14.21 \\
\hline 4187.039 & $\mathrm{Fe} \mathrm{I}$ & 2.450 & -0.550 & 47.95 \\
\hline 4187.795 & $\mathrm{Fe} I$ & 2.420 & -0.550 & 51.02 \\
\hline 4191.431 & $\mathrm{Fe} \mathrm{I}$ & 2.470 & -0.730 & 37.05 \\
\hline 4195.329 & $\mathrm{Fe} \mathrm{I}$ & 3.330 & -0.410 & 11.11 \\
\hline 4199.095 & $\mathrm{Fe} \mathrm{I}$ & 3.050 & 0.250 & 46.63 \\
\hline 4202.029 & $\mathrm{Fe} \mathrm{I}$ & 1.480 & -0.700 & 86.50 \\
\hline 4222.213 & $\mathrm{Fe} I$ & 2.450 & -0.970 & 30.57 \\
\hline 4227.427 & Fe I & 3.330 & 0.230 & 40.13 \\
\hline 4233.603 & $\mathrm{Fe} \mathrm{I}$ & 2.480 & -0.600 & 40.67 \\
\hline 4238.810 & Fe I & 3.400 & -0.230 & 19.09 \\
\hline 4250.119 & $\mathrm{Fe} \mathrm{I}$ & 2.470 & -0.400 & 50.75 \\
\hline 4260.474 & Fe I & 2.400 & -0.020 & 76.35 \\
\hline 4271.154 & Fe I & 2.450 & -0.350 & 57.11 \\
\hline 4282.403 & Fe I & 2.170 & -0.820 & 44.59 \\
\hline 4337.046 & $\mathrm{Fe} \mathrm{I}$ & 1.560 & -1.700 & 43.97 \\
\hline 4415.123 & $\mathrm{Fe} I$ & 1.610 & -0.610 & 85.44 \\
\hline 4430.614 & $\mathrm{Fe} \mathrm{I}$ & 2.220 & -1.660 & 12.55 \\
\hline 4442.339 & $\mathrm{Fe} \mathrm{I}$ & 2.200 & -1.250 & 27.52 \\
\hline 4447.717 & $\mathrm{Fe} \mathrm{I}$ & 2.220 & -1.340 & 25.53 \\
\hline 4461.653 & $\mathrm{Fe} \mathrm{I}$ & 0.090 & -3.200 & 60.88 \\
\hline 4466.552 & $\mathrm{Fe} \mathrm{I}$ & 2.830 & -0.600 & 30.33 \\
\hline 4489.739 & Fe I & 0.120 & -3.970 & 20.88 \\
\hline 4494.563 & Fe I & 2.200 & -1.140 & 35.54 \\
\hline 4528.614 & Fe I & 2.180 & -0.820 & 51.25 \\
\hline 4531.148 & $\mathrm{Fe} \mathrm{I}$ & 1.480 & -2.150 & 26.30 \\
\hline 4592.650 & Fe I & 1.560 & -2.460 & 13.65 \\
\hline 4602.940 & Fe I & 1.490 & -2.210 & 26.46 \\
\hline 4647.430 & Fe I & 2.950 & -1.350 & 4.34 \\
\hline 4871.318 & Fe I & 2.870 & -0.360 & 35.08 \\
\hline 4872.138 & $\mathrm{Fe} \mathrm{I}$ & 2.880 & -0.570 & 24.98 \\
\hline 4891.492 & $\mathrm{Fe} \mathrm{I}$ & 2.850 & -0.110 & 49.26 \\
\hline 4903.310 & Fe I & 2.880 & -0.930 & 14.44 \\
\hline 4918.994 & $\mathrm{Fe} \mathrm{I}$ & 2.870 & -0.340 & 36.83 \\
\hline 4920.503 & $\mathrm{Fe} \mathrm{I}$ & 2.830 & 0.070 & 55.96 \\
\hline 4938.814 & $\mathrm{Fe} I$ & 2.870 & -1.080 & 8.90 \\
\hline 4939.687 & Fe I & 0.860 & -3.340 & 14.13 \\
\hline 4994.130 & $\mathrm{Fe} \mathrm{I}$ & 0.920 & -3.080 & 20.67 \\
\hline 5001.864 & Fe I & 3.880 & 0.010 & 7.40 \\
\hline 5006.119 & Fe I & 2.830 & -0.620 & 23.36 \\
\hline 5041.072 & Fe I & 0.960 & -3.090 & 17.71 \\
\hline 5041.756 & Fe I & 1.490 & -2.200 & 24.04 \\
\hline 5049.820 & Fe I & 2.280 & -1.360 & 23.78 \\
\hline 5051.635 & Fe I & 0.920 & -2.800 & 32.09 \\
\hline 5068.766 & $\mathrm{Fe} \mathrm{I}$ & 2.940 & -1.040 & 7.63 \\
\hline 5079.740 & $\mathrm{Fe} \mathrm{I}$ & 0.990 & -3.220 & 12.20 \\
\hline 5083.339 & $\mathrm{Fe} \mathrm{I}$ & 0.960 & -2.960 & 22.72 \\
\hline 5110.413 & $\mathrm{Fe} \mathrm{I}$ & 0.000 & -3.760 & 41.65 \\
\hline 5123.720 & $\mathrm{Fe} \mathrm{I}$ & 1.010 & -3.070 & 17.15 \\
\hline 5127.359 & $\mathrm{Fe} I$ & 0.920 & -3.310 & 10.90 \\
\hline 5131.470 & Fe I & 2.220 & -2.520 & 2.60 \\
\hline 5254.955 & $\mathrm{Fe} I$ & 0.110 & -4.760 & 4.74 \\
\hline 5266.555 & $\mathrm{Fe} \mathrm{I}$ & 3.000 & -0.390 & 27.07 \\
\hline 5269.537 & Fe I & 0.860 & -1.320 & 106.40 \\
\hline 5281.790 & Fe I & 3.040 & -0.830 & 11.96 \\
\hline 5283.621 & $\mathrm{Fe} \mathrm{I}$ & 3.240 & -0.520 & 13.52 \\
\hline 5302.302 & Fe I & 3.280 & -0.880 & 7.25 \\
\hline 5307.361 & $\mathrm{Fe} \mathrm{I}$ & 1.610 & -2.990 & 4.02 \\
\hline
\end{tabular}


Table A.1. continued.

\begin{tabular}{rcrrr}
\hline \hline Wavelength $(\AA)$ & Species & L.E.P. $(\mathrm{eV})$ & $\log g f$ & $W_{\lambda}(\mathrm{m} \AA)$ \\
\hline 5324.179 & Fe I & 3.210 & -0.240 & 28.92 \\
5328.039 & Fe I & 0.920 & -1.470 & 97.30 \\
5328.532 & Fe I & 1.560 & -1.850 & 42.70 \\
5339.929 & Fe I & 3.270 & -0.720 & 9.95 \\
5367.470 & Fe I & 4.420 & 0.440 & 4.88 \\
5369.962 & Fe I & 4.370 & 0.540 & 8.29 \\
5371.490 & Fe I & 0.960 & -1.650 & 91.01 \\
5383.369 & Fe I & 4.310 & 0.640 & 15.37 \\
5393.168 & Fe I & 3.240 & -0.910 & 8.29 \\
5397.128 & Fe I & 0.920 & -1.990 & 78.19 \\
5405.775 & Fe I & 0.990 & -1.840 & 80.86 \\
5410.910 & Fe I & 4.470 & 0.400 & 4.34 \\
5415.200 & Fe I & 4.390 & 0.640 & 11.78 \\
5429.697 & Fe I & 0.960 & -1.880 & 82.44 \\
5434.524 & Fe I & 1.010 & -2.120 & 65.98 \\
5446.917 & Fe I & 0.990 & -1.910 & 77.60 \\
5455.609 & Fe I & 1.010 & -2.090 & 70.81 \\
5497.516 & Fe I & 1.010 & -2.850 & 26.69 \\
5501.465 & Fe I & 0.960 & -3.050 & 22.00 \\
5506.779 & Fe I & 0.990 & -2.800 & 29.13 \\
5569.618 & Fe I & 3.420 & -0.540 & 10.23 \\
5572.842 & Fe I & 3.400 & -0.310 & 15.23 \\
5576.089 & Fe I & 3.430 & -1.000 & 3.27 \\
5586.756 & Fe I & 3.370 & -0.140 & 21.36 \\
5615.644 & Fe I & 3.330 & -0.140 & 30.07 \\
6065.480 & Fe I & 2.610 & -1.410 & 9.84 \\
6136.615 & Fe I & 2.450 & -1.400 & 17.43 \\
6137.692 & Fe I & 2.590 & -1.400 & 12.11 \\
4233.172 & Fe II & 2.580 & -1.900 & 36.10 \\
4416.830 & Fe II & 2.780 & -2.410 & 7.66 \\
4491.405 & Fe II & 2.860 & -2.700 & 7.20 \\
4508.288 & Fe II & 2.860 & -2.250 & 13.71 \\
4515.339 & Fe II & 2.840 & -2.450 & 9.79 \\
4520.224 & Fe II & 2.810 & -2.600 & 8.70 \\
4522.634 & Fe II & 2.840 & -2.030 & 21.74 \\
4541.524 & Fe II & 2.860 & -2.790 & 4.39 \\
4555.893 & Fe II & 2.830 & -2.160 & 13.09 \\
4576.340 & Fe II & 2.840 & -2.820 & 3.84 \\
5234.625 & Fe II & 3.220 & -2.150 & 11.24 \\
5476.900 & Ni I & 1.830 & -0.890 & 40.62 \\
4722.153 & Zn I & 4.030 & -0.338 & 3.42 \\
4810.528 & Zn I & 4.080 & -0.137 & 3.57 \\
\hline & & & & \\
5 & & & \\
5 & & & \\
5 & & &
\end{tabular}

